Published in: Composite Structures

Accepted: 15 September 2013 (approximate date)

DOI: https://doi.org/10.1016/j.compstruct.2020.111916

\title{
Strain and damage monitoring in CFRP fuselage panels using fiber Bragg grating sensors. Part I: Design, manufacturing and impact testing
}

\author{
K.I. Tserpes ${ }^{\mathrm{a}, *}$, V. Karachalios ${ }^{\mathrm{b}}$, I. Giannopoulos ${ }^{\mathrm{b}}$, V. Prentzias ${ }^{\mathrm{b}}$ and R. Ruzek \\ ${ }^{a}$ Laboratory of Technology \& Strength of Materials, Department of Mechanical Engineering \& \\ Aeronautics, University of Patras, Patras 26500, Greece \\ ${ }^{\mathrm{b}}$ Hellenic Aerospace Industry, Research and Development Department, 32009 Schimatari, \\ Greece \\ 'Výzkumný a zkušebni letecký ústav, a.s. (VZLÚ), Beranových 130, 19905 Prague 9 - Letnany, \\ Czech Republic
}

\begin{abstract}
:
This is the first paper of a two-paper series describing design, implementation and validation of a strain and damage monitoring system for CFRP fuselage stiffened panels based on fiber optic Bragg grating sensors. The monitoring system was developed and tested on the basis of three load-scenarios: compression to failure of the undamaged panel, compression to failure of the impacted panel and compression to failure of the impacted and fatigued panel. This paper focuses on the design of the fuselage panel, the design of the monitoring system, the embedment of fiber sensors in the panel during manufacturing and the impact testing. The network of the sensors was designed based on a numerical buckling analysis from which the strain field of the panel was computed as a function of the applied compressive load. Embedment of fiber sensors in the panel was done so as to minimize risk of fiber breaking during manufacturing and impact testing and to effectively capture strains that are representative of damage developed in the panel due to compressive load. Barely visible and visible low velocity impact damage sites were created at different locations of the panel using a drop-weight impactor. The panels were inspected using C-scan just after manufacturing, to check quality of the material, and just after impact testing to detect impact damage at each location.
\end{abstract}

Keywords: Structural health monitoring, CFRP panels, Fiber-optic Bragg gratings, Buckling, Impact, Optimization.

*Corresponding author. Tel.:+30-2610-969498, Fax:+30-2610-997190, e-mail:kit2005@mech.upatras.gr 


\section{Introduction}

Fiber-reinforced polymer composites are revolutionizing the design of large, highperformance structures in the aerospace industry. The use of carbon fiber-reinforced plastics (CFRPs), the most common and lightest of industrial composite materials, is now very widespread; for instance, they comprise almost $50 \%$ of the new Boeing 787 Dreamliner and more than $20 \%$ of the Airbus A380. The extended use of CFRPs in fuselage structures has increased the need for the use of non-destructive testing techniques to detect damage that could counterbalance the integrity of composite structures. In the last few years, research was devoted to the development of structural health monitoring (SHM) systems, which are one step ahead from conventional non-destructive testing systems as they offer online monitoring of the structures.

SHM systems realize continuous monitoring of structure's integrity through strategically located sensors coupled with monitoring technology enabling remote interrogation. There are a variety of available approaches. The most common are strain based SHM systems which measure the strain distribution in the structure subject to operational loads via electrical resistive strain gauges or optical fiber sensors. Any damage in the structure, that causes a change in the strain distribution, may be potentially detected by this type of system. Fiber optic sensors are particularly suitable for the health monitoring of large structures, such fuselage panels, because multiple sensors can be attached to a single fiber for distributed sensing over long distances. The small size of fiber optic sensors ( $<250$ micron in diameter) imposes negligible intrusion into the host structure and allows fast interrogation with minimal wiring requirements. The most widely used fiber optic sensor is the Fiber Optic Bragg Grating (FOBG) sensor. This sensor possess superior characteristics such as localized strain measurement ability, relatively small size, high sensitivity, inertness to electric or magnetic inference and multiplexing capability. A fundamental issue in the implementation of a FOBG-based SHM system in composite structures is the embedment of sensors during manufacturing. Although manufacturing methods of composites parts offer the opportunity to build the sensors into the part itself, there are several difficulties to overcome such as the selection of points of ingress and egress of sensor data lines and the possible effect of sensors and ancillaries on the mechanical properties of the composite.

Although major aircraft manufacturers, both civil and military, are keen to introduce SHM systems into future aircrafts, particularly in composite structures, to the date, SHM systems were successfully installed only in experimental applications. In most of these applications FOBG-based SHM systems were used [1-23]. In an early work, Takeda et al 
[7] have managed to relate governed delamination onset and growth in CFRP laminates with the spectrum of FOBGs. In a latter work, Yashiro and Okabe [20] implemented the methodology proposed in [7] for holed laminates subjected to static and fatigue load. The most important finding of [20] is that fatigue damage in the composite material may not be detected by FOBGs due to debonding of the sensors caused by the cyclic loading. This finding is further analyzed and discussed in [21].

In the majority of reported works, FOBGs were used to detect damage in specimens. There are very few works in which FOBGs were embedded in structural parts $[10,17,21,23]$. Tsutsui et al. in [10] applied small-diameter optical fiber sensors to stiffened composite panels for the detection of impact damage. Ryu et al. [17] have used multiplexed and multi-channeled built-in FOBGs to monitor the buckling behavior of a composite wing box. Recently, Takeda et al. [23] used FOBG sensors to monitor damage due to compressive load in CFRP stiffened panels.

In the present work, an integrated methodology for monitoring strain and damage in CFRP fuselage panels was designed, implemented and validated on the basis of a series of load-cases representing critical scenarios for the CFRP structure. The design of the flat panel was based on a structural optimization performed for a curved stiffened panel which is part of a composite fuselage barrel. The sensors' network was designed based on a buckling analysis of the panel conducted using explicit FEA. Embedment of fiber sensors in the panel during manufacturing was done so as to minimize risk of fiber breaking during manufacturing and impact testing and to effectively capture strains being representative of the damage developed in the panel. Low velocity impact damage of two types namely, barely visible impact damage and visible impact damage was created at different locations of the panel using a drop-weight impactor. Finally, three different panels were mechanically tested to validate the proposed monitoring system. This paper, which is the first of two-paper series, describes the design of the panel, the monitoring system design, the numerical analysis, the manufacturing of the panel and the impact testing. The second paper [24] describes mechanical testing and validation of the proposed strain and damage monitoring system.

\section{Design of the CFRP panel}

To design the flat panel (i.e. geometry and materials), a numerical structural optimization was performed aiming to create the lightest possible panel that fulfils the structural design criteria set along with any other type of constraints need to be met by the structure. The structural optimization was performed for a curved panel which is a part of a fuselage 
barrel and then applied to the flat panel considered for the development of the strain and damage monitoring system. In this section, the methodology and results of the structural optimization are described.

\subsection{Formulation of the optimization problem}

The particular structural optimization problem is described by a number of design variables and design constraints. A multi variable optimization problem is strongly affected by the solution strategy and algorithm considered. Moreover, it can be time consuming and the final design solution set achieved can be questioned if it is indeed the "global" optimum variables set or not. For this reason, special effort was placed to diminish the number of design variables and constraints in this problem. Another peculiarity of similar type of optimization problems is that most of the design variables are discrete, i.e. not continuously ranging inside a real number range, meaning, that the optimized number of applied stiffeners, for example must be an integer value e.g. 4, 5, 12 stiffeners, etc and not a real one e.g. 7.56 stiffeners. The same holds for most of the design variables considered in the present work. Apart from the discrete variable optimization problem, there are also certain manufacturing and design constraint rules that need to be fulfilled. These rules refer to product realization and are not relevant to the optimization algorithm itself. The design constraint rules were transformed into design value space boundaries for the design variables.

\subsection{The structural design criteria}

The stiffened curved panel was designed on the basis of a combined compression/shear load. The structural design criteria were:

- The panel should endure ultimate load without failure,

- The panel should not fail due to global buckling instability at limit load. Local buckling is permitted as long as the post buckled shape does not induce failure strains.

\subsection{Design variables and constraints}

A great effort was placed in the reduction of the number of design variables. Based on information from $[25,26]$, for similar stiffened panel geometries, the omega shape was chosen for the stringers. Design variables corresponding to dimensions of the stringer, shown in Fig.1, were considered as predefined. 
The range of skin thickness at the bays and the stringers was preset to $1 \mathrm{~mm}$ to 2.5 $\mathrm{mm}$. This configuration follows the rules of a symmetrical and balanced layup. The applicable layup configuration for the above mentioned range and specifically for the stringers was divided into three categories: stiff, medium and soft layup. These layups are characterized as such according to the $0^{\circ}$ ply content. Implementation of predetermined constraints in the problem corresponds to the effective translation of design variables into a series of applicable layup configurations of thickness ranging from $1 \mathrm{~mm}$ to $2.5 \mathrm{~mm}$ and a stiff-medium-soft categorization with respect to the longitudinal modulus of the stringers.

The final optimization variables and the respective constraints chosen for the algorithm were:

- The number of stringers (ranging from 24 to 40 for the full barrel geometry)

- The layup of the stringers (stiff, medium, soft)

- The skin thickness at the stiffeners $(1 \mathrm{~mm}-2.5 \mathrm{~mm})$

- The skin thickness at the bays $(1 \mathrm{~mm}-2.5 \mathrm{~mm})$

\subsection{Optimization algorithm}

The optimization algorithm was based on an iterative neighbouring point search (selection of best design set) according to which for a given set of design variables, the effect upon the non-linear behavior of the panel due to compression and shear was evaluated using FEA by considering also the mass of the part that corresponds to the specific set of variables. For the neighboring set of variables the response was also evaluated using FEA and the route to optimization was decided based on the effectiveness of the response correlated to the mass of the part. The loading conditions for the curved panel were computed from FEA of the complete barrel subjected to combined compression and shear. FE mesh of the barrel is shown in Fig.2.

\subsection{Optimization results}

The optimization results are presented in the graphs of Fig. 3 which plot the design variables with regard to optimization cycles. From each analysis the minimum design variable was selected at it leads to the minimum mass of the panel. The optimum panel configuration has 24 stringers with a thickness of $2 \mathrm{~mm}$, the medium (2) layup and a skin with a thickness of $2 \mathrm{~mm}$. The thickness at the other parts of the barrel, i.e. stringer foot and stiffener is not $2 \mathrm{~mm}$. Fig. 4 shows the variation of thickness in the FE model of the curved barrel used for the structural optimization. The same configuration was also used 
for the flat panel. The only difference is in the total number of stringers which was selected by following the analogy of number of stringers per panel length derived for the curved panel. The dimensions of the optimum stringers, with reference to Fig.1, are: $\mathrm{a}=31.75 \mathrm{~mm}, 25.7 \mathrm{~mm}, \mathrm{c}=18.18 \mathrm{~mm}$ and $\mathrm{d}=50^{\circ}$.

\section{Geometry, materials and loading conditions of the flat panel}

Consider the flat CFRP panel schematically shown in Fig.5. The panel is stiffened by omega stringers, shown in Fig.1, and contains also three aluminum frames. The panel is made from the unidirectional HexPly 8552 (by Hexcel) prepreg composite material. This is a high performance tough epoxy matrix prepreg for use in primary aerospace structures. It exhibits good impact resistance and damage tolerance for a wide range of applications. The layup of the skin was $\left[45^{\circ} / 90^{\circ} /-45^{\circ} / 0^{\circ} / 45^{\circ} / 90^{\circ} /-45^{\circ} / 0^{\circ}\right]_{S}$ and that of the stringers was $\left[45^{\circ} /-45^{\circ} / 0^{\circ}{ }_{2} / 90^{\circ} / 0^{\circ}\right] \mathrm{s}$.

The scope of the present work was to develop a system based on FOBGs capable for monitoring strain and damage of the panel under loading scenarios that are representative of in-service loading conditions. In this frame, following loading scenarios were chosen:

1. Compression to failure of undamaged panel (panel_1),

2. Compression to failure of panel with barely visible impact damage (BVID) after fatigue (panel_3), and

3. Compression to failure of panel with visible impact damage (VID) (panel_3). The specific loading conditions were chosen because they are highly prospective for a bottom fuselage panel. Moreover, it is known that compression after impact represents the "Achilles' heel" of composite panels. The BVID and VID locations were determined so as to influence the overall panel's performance. The external dimensions of the panel as well as the locations of BVID and VID sites are indicated in the schematic of Fig.6.

To consider the effect of initial damage, which is a very often critical phenomenon in composite materials, artificial delaminations of three different sizes were inserted at specific locations of panel_2. Artificial delamination "A" of size $25 \mathrm{~mm}$ x $12 \mathrm{~mm}$ was inserted between the stringer foot and the skin, artificial delamination "B" of size $12 \mathrm{~mm}$ x $12 \mathrm{~mm}$ was inserted between the skin and the corner of the stringer and artificial delamination " $\mathrm{C}$ " of size $25 \mathrm{~mm}$ x $25 \mathrm{~mm}$ was inserted in the skin between the $2 \mathrm{nd}$ and the 3rd bottom layers. The locations of artificial delamination are indicated in the schematic of Fig.7. Each artificial delamination was inserted into two positions, thus creating in total six sites of artificial delamination in the panel. 


\section{Monitoring system}

To design, implement and validate the monitoring system, the following steps were undertaken:

1. Design of the CFRP flat panel based on the structural optimization of a curved panel which is a part of a fuselage barrel,

2. Numerical modeling of the panel's compressive behavior and computation of the strain field in the panel as a function of applied load to indentify strains related to critical damage,

3. Design of the fiber sensors' network based on the number of available sensors, the requirements for strain measuring and topology of impact locations,

4. Manufacturing of the panels and embedment of fiber sensors in the panel,

5. Ultrasound $\mathrm{C}$-scan testing of the panel to check manufacturing quality,

6. Impact testing to create multi-site BVID and VID impact damage in the panel,

7. Mechanical testing to evaluate the capability of the system to monitor the strain and damage in the panels.

In the following sections, steps 1 to 6 of the process will be described. Step 7 is described in [24].

\section{Numerical analysis}

In order to properly design the fiber sensors' so as to effectively measure strains, which are representative of the critical damage that will be developed during compression of the panel, explicit FEA of the panel was performed. FEA is also expected to assist the design of mechanical testing as it will lead to correlation between the applied load and damage progression based on which the load sequence in the experiment will be determined.

Explicit FEA was performed using the LS-DYNA commercial FE code [27]. To this end, a 2D FE model of the panel was created by importing into the ANSYS preprocessor the CATIA drawing shown in Fig.5. The entire panel was represented using the ANSYS SHELL63 element. This is an explicit thin structural element which has also a composite layered option. SHELL163 has both bending and membrane capabilities and permits both in-plane and normal loads. The FE mesh of the panel is depicted in Fig.8. Through the thickness of the skin and the stringer four and one elements were adopted, respectively. This was led to the modeling of 4 layers per element for the skin and 12 layers per element for the stringer.

The boundary conditions applied to the model of the panel were identical to those applied in the tests. They comprised the application of compressive load, which was 
realized through two epoxy potting frames mounted at the two sides of the panel, and the application of the anti-buckling device which preserves lateral global buckling of the panel. In the model, the compressive load was modeled by restraining the DOFs of the nodes that are within the area covered by the potting frame: at one side (steady side) all DOFs were restrained and at the opposite side (moving side) all DOFs were restrained except from the longitudinal translation which was set equal to an axial incremental compressive displacement. Also, the nodes that fall within the anti-buckling device had all their DOFs, except from longitudinal translation, restrained. A schematic of the boundary conditions applied to the model is given in Fig.9.

The numerical load-displacement curve of the panel is compared with the experimental curve from [24] in Fig.10. Between the model and test, a very good agreement was obtained both in terms of stiffness and maximum load. The model predicted a sequence of buckling modes in the bays. Local buckling initiated around 70 $\mathrm{kN}$ as indicated in the contour of normal displacement and deformed shape of the panel illustrated in Fig.11(a). In Fig.11(a), buckling of the bays is also visible. From the values of normal displacement in Fig.11(a), it becomes evident that buckling is more severe at the bays close to the loaded side. With increasing load, buckling of the skin became more severe, by changing modes, and the amount of load that was transferred by the stringers increased. Final failure of the panel predicted at $475 \mathrm{kN}$ due to (global) buckling of the middle stringer developed close to the loaded side after the first metallic frame. The predicted contour of normal displacement and deformed shape of the panel at $475 \mathrm{kN}$ are shown in Fig.11(b). The fact that both numerical and experimental load-displacement curves are almost linear reveals that local buckling did not cause any significant damage in the panel.

\section{Manufacturing of the panel}

\subsection{Design of sensors' network}

Based on the numerical results and the number of available FOBG sensors, the sensors' network was designed so as to enable measurement of the strains that are related to the basic damage patterns developed in the panel during loading. As described in Section 5, there are two main damage modes: buckling in the bay, which takes place progressively at various modes and buckling of the stringers, which causes final failure of the panel. Therefore, it is very crucial to effectively measure the strains in the bay and being capable to relate their extreme values with buckling initiation and alteration of buckling modes. 
Moreover, strains at the stringers must be also measured in order to enable monitoring of buckling initiation which causes catastrophic failure of the panel.

At each of the three panels, nine FOBGs were mounted following a configuration of three sensors per line. Positioning networks of the sensors in the panel are shown in Fig.12, while Table 1 demarcates the sensors for each panel. The sensors were placed at the middle section of the bays as well as at the edge of the stringers. In panel_1, the sensors were bonded externally. In panel_1 and panel_2 the sensors were placed at the bays of the same side with respect to the axis of the panel, while in panel3 the sensors were placed at antisymmmetric bays. In panel_2, the sensors placed at the middle of the bays are very close to the artificial delamination as well as to the impact damage sites. Thus, they are expected to capture any phenomena developed there, due to the concentration of initial damage, by recording extreme values of strain.

To be able to validate the measurements given by the FOBGs, a large number of strain gauges were mounted externally to the panel. Besides validation of the FOBGs, the strain gauges will offer a global view of the strain field in the panel which is very crucial for the thorough understanding of the panel's mechanical behavior. Information about the exact number and placement of strain gauges are given in [24].

\subsection{Embedding procedure}

Embedment of FOBG sensors during manufacturing is a tedious task since it involves many difficulties such as the selection of ingress and egress points of sensor data lines, the possible effect of sensors and ancillaries on the mechanical properties of the composite and the risk of sensor breaking during fabrication. The main idea behind the process that was adopted is that FOBG lines will be "emerging" from the composite surface (inside the part boundary) and not from the edges of the part. This is because in every composite manufacturing process a part trimming operation (all around the boundary of the part) is usually required after curing. Therefore, if the FOBG lines were placed to "emerge" from the edges of the part, they would be destroyed during the trimming operation.

The FOBG sensors used in the present study were supplied by FiberSensing (http://www.fibersensing.com) in arrays of three sensors. The wavelengths of the sensors at each array were $1526 \mathrm{~nm}, 1547 \mathrm{~nm} \& 1568 \mathrm{~nm}$, respectively. Due to the intense temperature/pressure conditions occuring during curing the primary coating of the optical fiber was polyimide with polyimide recoating on the grating location after inscription of FOBGs in order to improve mechanical performance. The FOBG lines were embedded 
"bare" (without any buffer protection) so that there is direct strain transfer from the laminate to the FOBGs. Only at the position of ingress/egress points a small length of buffer cable (typ. $0.4 \mathrm{~mm}$ in diameter - also polyimide) was placed for extra protection of the fiber. The embedding procedure was implemented by means of the following steps:

- Building of the layup of the prepreg material Hexply 8552 in UD configuration disposed in a lower and upper stack (in respect to the position that the FOBG line will be placed).

- Use of a rubber disk suitable to be bonded on the prepreg material surface (upper stack) in the polymerization cycle (in order to protect the optical fiber at the ingress/egress points).

- Place lower stack on mould.

- "Piercing" the FOBG line through the upper stack layers by using a hollow needle (pierce also through the rubber disks).

- Remove the needles and gently stretch and bond (using small quantities of spray adhesive) the FOBG line on the bottom surface of the upper stack.

- Place the upper stack on top of the already positioned bottom stack (thus embedding the FOBG line between the upper and lower stack) and perform a debulking process.

- Isolate the FOBG lines from the laminate surface by enclosing them in standard release film so as to protect the FOBG lines from the resin float that will occur during the polymerization cycle. This way the FOBG line will remain clean from excess resin after the curing cycle and will be possible to apply a connector by standard splicing technique.

- Complete the standard autoclave bagging process as usual (use of peel plies, breather, vacuum bags etc)

Fig. 13 shows the configuration of a sensor data line connecting the sensors at the middle of the bay and at the edge of the stringer as well as a close view of the ingress and egress points. In both cases (skin \& stringer) the sensors were placed in the middle of the laminate thickness between the consecutive $0^{\circ}$ layers. FC/APC connectors were placed on the fiber optic cables by the standard splicing process.

The FOBGs were mounted externally in panel_1 using the following two-step process:

1. First, the part of the fiber optic containing the Bragg Grating was bonded by cyanoacrylate adhesive on the selected position on the panel, and 
2. Then, a slightly larger length of the fiber optic (including the FOBG) was covered with standard two part epoxy paste adhesive (Araldite ${ }^{\circledR} 2015$ by HUNTSMAN) for extra protection and bonding security.

\section{Non-destructive inspection}

The panels were inspected by C-scan twice: once after manufacturing to check the quality of the fabricated composite material and also for possible initial damage due to the presence of the sensors and once after being impacted to check the extent of impact damage created. The type of inspection was immersion Pulse/Receive on the entire part with A-Scan and C-Scan data acquisition and presentation. Inspection was performed by a UT-Level 1 Inspector. The equipment used to conduct the tests comprises:

- UT equipment (Bridge type, 3 axis immersion tank),

- Pulser/Receiver PR-90,

- Analog/Digital AD-IPR-1210,

- UT Probe Mateval 5Mhz (focus) - 20mm diameter,

- Ultrasonic couplant Water, and

- Post Processing Software UTIA 4.1 Professional by Mistras Group Hellas.

Calibration was performed on predetermined and recorded specific flat areas ranging from $20-30 \mathrm{~mm}$ to $280-300 \mathrm{~mm}$ from the panel edge. Calibration was repeated at the end of the scanning of all panels to ensure accurate readings.

The C-scan graphs taken just after manufacturing of the panels are depicted in Fig.14. The graphs show a very good material quality with no manufacturing defects for all three panels. In the C-scan of panel_1 (Fig.14a), there is no initial delamination at the stringer to the skin attachment area or the entire skin; the only visible areas in the scan are the hi-lock fastener holes opened to assemble the frames. In the C-scan graph of panel_2 (Fig.14b), the visible areas are more due to the presence of artificial delaminations and the sensors. The numbered visible areas on the graph of panel_2 (Fig.14b) indicate: (1) artificial delaminations, (2) ingress points of FOBGs in the skin, (3) FOBG embedded in the skin and (4) fastener holes. Similarly, in the C-scan of panel_3 (Fig.14c) the numbered visible areas on the graph indicate: (2) the entry points of FOBG's at skin, (3) part of FOBG embedded in the skin, (4) fastener holes, (5) light mould marks at back side of stringers, and (6), the point of connection between stringers. C-scan graphs of the impact damage sites will be presented in the next section.

\section{Impact testing}


Impact damage was created in panel_2 and panel_3 using an INSTRON Dynatup 9250 HV drop tower. Several experiments were conducted to create BVID and VID sites at the specified locations indicated in Fig.6 using a semi-circular impactor with a diameter of $16 \mathrm{~mm}$ (Fig.15). During testing force and energy versus time and deflection were recorded. A full lateral support at the large sides of the panels was applied during impact. To apply these boundary conditions, the special metallic device shown in Fig.16 was built.

Initially, panel_3 with BVID was tested. BVID is defined as the indentation of 0.8$1 \mathrm{~mm}$ or cut-off energy of $50 \mathrm{~J}$. Due to the unknown impact behavior of the panel, BVID was inclined at each position of the panel by performing repeated impacts with very small energies up to the point where the created impact damage falls within the definition of the BVID. The number of repeated impacts and their impact energy were decided based on the energy-time curve of the first impact and the observed initial impact damage. In Fig.17, the variation of impact energy with respect to the deflection is plotted for the BVID1, BVID2 and BVID3 locations. The plots for the remaining locations are not shown for the sake of briefness. In the case of BVID1 (Fig.17a), four impacts of large energy were necessary to create the desired impact damage. This is because this location lies at the middle of the bay where the panel has smaller local rigidity and a large part of the skin is free to vibrate, thus leading to the release of a considerable amount of energy. However, this is not the case for BVID2 (Fig. 17b) and BVID3 (Fig.17c), which lie on the stringer and at the edge stringer, respectively. For these two locations the entire impact energy was absorbed by the panel. Fig. 18 shows a close view of the damage developed at BVID5 as well as the $\mathrm{C}$-scan of this area showing the extent of delamination and matrix cracking created due to impact.

The variation of energy with respect to the deflection for the VID1, VID2 and VID3 locations is plotted in Fig.19. In this case, much larger impact energies were applied as practically there is no threshold for the developed impact damage. In most VID tests, a hole was created in the panel. This is also the case for VID4 shown in Fig.20. The C-scan of the impacted area (Fig.20c) shows the amount of delamination and matrix cracking accumulated around the hole.

\section{Conclusions}

In the present paper, the first part of the work conducted to develop a methodology for monitoring strain and damage in CFRP fuselage panels based on FOBGs is described. Design, implementation and validation of the monitoring system were done on the basis 
of a series of load-cases representing critical scenarios for the CFRP panel. The content of the paper can be summarized as follows:

1. The flat CFRP panel was designed based on a structural optimization performed on a similar curved panel which is a part of a fuselage barrel.

2. The sensors' network was designed on the basis of a buckling analysis of the panel conducted using explicit FEA. Numerical results reveal the need for the sensors to capture two main damage modes: early buckling of the skin with varying modes and latter buckling of the stringer which causes final failure of the panel. To meet this requirement, nine sensors were mounted on the panels at the middle of the bays and at the edges of the stringers.

3. Embedment of fiber sensors in the panel during manufacturing was done so as to minimize risk of fiber breaking during manufacturing, and also minimize the amount of damage created in the composite material by the sensors. C-scan tests conducted just after fabrication of the panel showed a very good material quality and a minor damage at the points of sensors' ingress and egress. Validation of the effectiveness of the method used to embed the sensors in the panels will be completed by the evaluation of the results from mechanical tests [24].

4. Using a drop-weight impactor impact damage of BVID and VID was created at various sites in the panels. A second row of C-scan tests were conducted to check the amount of damage developed due to impact.

In the second paper [24], mechanical testing and validation of the proposed strain and damage monitoring methodology is described.

\section{Acknowledgment}

The research leading to these results has received funding from the European Community's Seventh Framework Programme (FP7/2007-2013) for the Clean Sky Joint Technology Initiative under grant agreement $n^{\circ}$ CSJU-GAM-GRA-2008-001 and grant agreement $n^{\circ} 296514$.

\section{References}

[1] Huang S, Ohn MM, Leblanc M, Measures RM. Continuous arbitrary strain profile measurements with fiber Bragg gratings. Smart Mater Struct 1998;7(2):248-56.

[2] Rippert L, Wevers M, Huffel SV. Optical and acoustic damage detection in laminated CFRP composite materials. Compos Sci Technol 2000;60(14):2713-24.

[3] Lau K-T, Yuan L, Zhou L-M, Wu J, Woo C-H. Strain monitoring in FRP laminates and concrete beams using FBG sensors. Composite Structures 2001;51:9-20. 
[4] Hong CS, Ryu CY, Lee JR, Kim CG. Buckling behavior monitoring of composite wing box model using fiber Bragg grating sensor system. Proc SPIE 2001;4327:6608 .

[5] Jones R, Galea S. Health monitoring of composite repairs and joints using optical fibers. Composite Structures 2002;58(3):397-403.

[6] Zhao Y, Ansari F. Embedded fiber optic sensor for characterization of interface strains in FRP composite. Sensors Actuators A: Phys 2002;100(2-3):247-51.

[7] Takeda S, Okabe Y, Takeda N. Delamination detection in CFRP laminates with embedded small-diameter fiber Bragg grating sensors. Composites Part A 2002;33(7):971-80.

[8] Baker W, McKenzie I, Jones R. Development of life extension strategies for Australian military aircraft, using structural health monitoring of composite repairs and joints. Composite Structures 2004;66:133-143.

[9] Ling H-Y, Lau K-T, Cheng L. Determination of dynamic strain profile and delamination detection of composite structures using embedded multiplexed fibre-optic sensors. Composite Structures 2004;66(1-4):317-26.

[10] Tsutsui H, Kawamata A, Sanda T. and Takeda N. Detection of impact damage of stiffened composite panels using embedded small-diameter optical fibers. Smart Mater. Struct. 2004;13:1284-1290.

[11] McKenzie I, Jones R, Marshall IH, Galea S. Optical fibre sensors for health monitoring of bonded repair systems. Composite Structures 2000;50:405-416.

[12] Wevers M, Rippert L, Papy JM, Huffel SV. Processing of transient signals from damage in CFRP composite materials monitored with embedded intensity modulated fiber optic sensors. NDT E Int 2006;39(3):229-35.

[13] Tsuda H. Ultrasound and damage detection in CFRP using fiber Bragg grating sensors. Compos Sci Technol 2006;66(5):676-83.

[14] Frieden J, Cugnoni J, Botsis J, Gmür T. Low energy impact damage monitoring of composites using dynamic strain signals from FBG sensors - Part I: Impact detection and localization. Composite Structures 2012;94:438-445.

[15] Frieden J, Cugnoni J, Botsis J, Gmür T. Low energy impact damage monitoring of composites using dynamic strain signals from FBG sensors - Part II: Damage identification. Composite Structures 2012;94:593-600.

[16] Ling H-Y, Lau K-T, Su Z, Wong E T-T. Monitoring mode II fracture behaviour of composite laminates using embedded fiber-optic sensors. Compos Part B 2007;38: 488-497.

[17] Ryu C-Y, Lee J-R, Kim C-G, Hong C-S. Buckling behavior monitoring of a composite wing box using multiplexed and multi-channeled built-in fiber Bragg grating strain sensors. NDT\&E International 2008;41:534-543.

[18] Li F, Murayama H, Kageyama K, Shirai T. Doppler effect-based fiber-optic sensor and its application in ultrasonic detection. Opt Fiber Technol 2009;15(3):296-303.

[19] Fu T, Liu Y, Li Q, Leng J. Fiber optic acoustic emission sensor and its applications in the structural health monitoring of CFRP materials. Opt Lasers Eng 2009;47(10):1056-62.

[20] Yashiro S, Okabe T. Estimation of fatigue damage in holed composite laminates using an embedded FBG sensor. Composites Part A 2011;42:1962-1969.

[21] Minakuchi S, Takeda N, Takeda S, Nagao Y, Franceschetti A, Liu X. Life cycle monitoring of large-scale CFRP VARTM structure by fiber-optic-based distributed sensing. Composites Part A 2011;42(6):669-76.

[22] Papantoniou A, Rigas G, Alexopoulos ND. Assessment of the strain monitoring reliability of fiber Bragg grating sensor (FBGs) in advanced composite structures. Composite Structures 2011;93:2163-2172

[23] Takeda S. Aoki Y, Nagao Y. Damage monitoring of CFRP stiffened panels under compressive load using FBG sensors. Composite Structures 2012;94:813-819.

[24] Ruzek R, Kudrna P, Kadlec M, Karachalios V, Tserpes KI. Strain and damage monitoring in CFRP fuselage panels using fiber Bragg grating sensors. Part II: Mechanical testing and evaluation. submitted to Composites Part Structures 2013. 
[25] Kim G-H, Choi J-H, Kweon J-H. Manufacture and performance evaluation of the composite hat-stiffened panel. Composite Structures 2010;92:2276-2284.

[26] Mittelstedt C, Beerhorst M. Closed-form buckling analysis of omega-stringerstiffened composite panels considering periodic boundary conditions. Composite Structures 2009;88:424-435.

[27] ANSYS Mechanical/LS-DYNA, Release 11.0. 


\section{Captions to Figures}

Fig.1: Schematic and dimensions of the omega stringer.

Fig.2: $\quad$ FE mesh of the fuselage barrel.

Fig.3: Variation of design variables with respect to design cycles: a. number of stringers, stringer layup, stringer thickness, skin thickness.

Fig.4: Variation of skin thickness in the FE model of the curved barrel.

Fig.5: Schematic of the flat CFRP stiffened panel.

Fig.6: Schematic of the panel including dimensions, locations of impact damage and artificial delamination.

Fig.7: Schematic illustrating locations of artificial delaminations.

Fig.8: $\quad$ FE mesh of the flat panel used for buckling analysis.

Fig.9: Schematic of the boundary conditions applied to the model of the panel.

Fig.10: Comparison between numerical and experimental [24] load-displacement curve of the panel.

Fig.11: Predicted contour of normal displacement (in $\mathrm{mm}$ ) and deformed shape of the panel at a. $70 \mathrm{kN}$ and b. $475 \mathrm{kN}$.

Fig.12: Positioning of the sensors in the panel.

Fig.13: a. Configuration of sensor data line, $b$. Close view of the sensor ingress point.

Fig.14: C-scan graphs of the panels taken after manufacturing: a. panel_1, b. panel_2, and c. panel_3.

Fig.15: Photo of the impactor above BVID5 location.

Fig.16: Photo of the panel supported by the metallic device just before subjected to impact testing.

Fig.17: Plots of energy versus deflection recorded during a. BVID1 test, b. BVID2 test and $\mathrm{c}$. BVID3 test.

Fig.18: a. Close view of the impact damage at BVID5 location and b. C-scan of the area around BVID5 location.

Fig. 19: Plot of energy versus deflection recorded during VID tests.

Fig.20: Hole created due to impact at VID4 location: a. impacted side and b. opposite side. c. C-scan around VID4 location. 
Fig.1

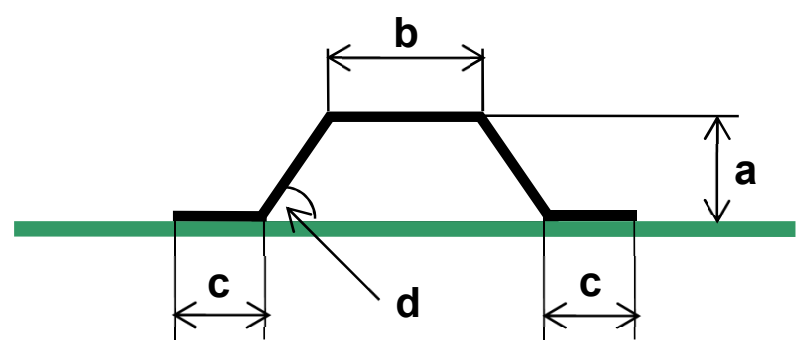

a: stringer's height

b: stringer's upper cap width

c: stringer's lower feet width

d: angle of stringer's webs 
Fig.2

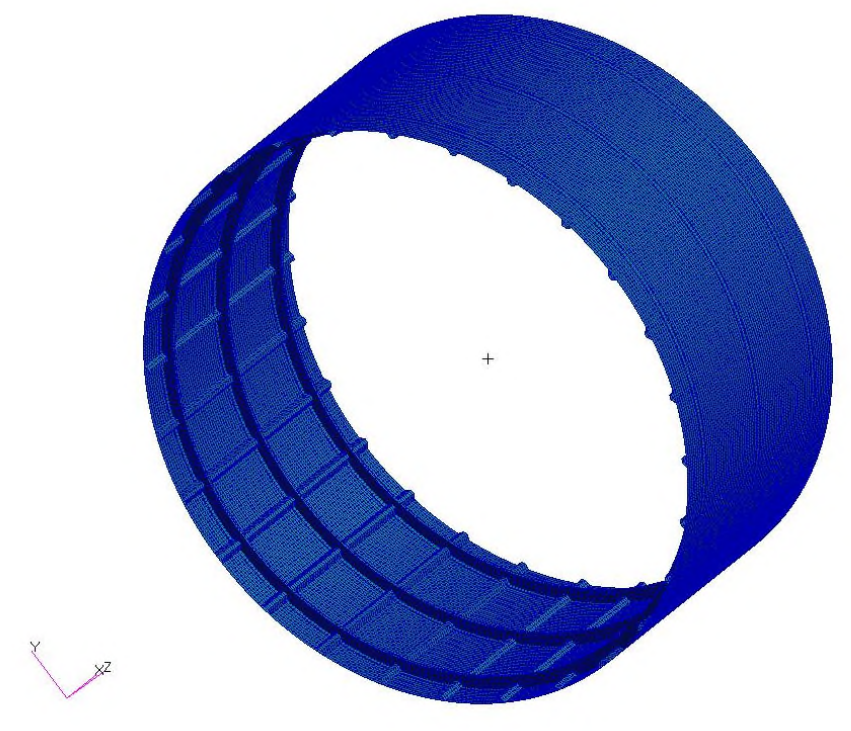


Fig.3
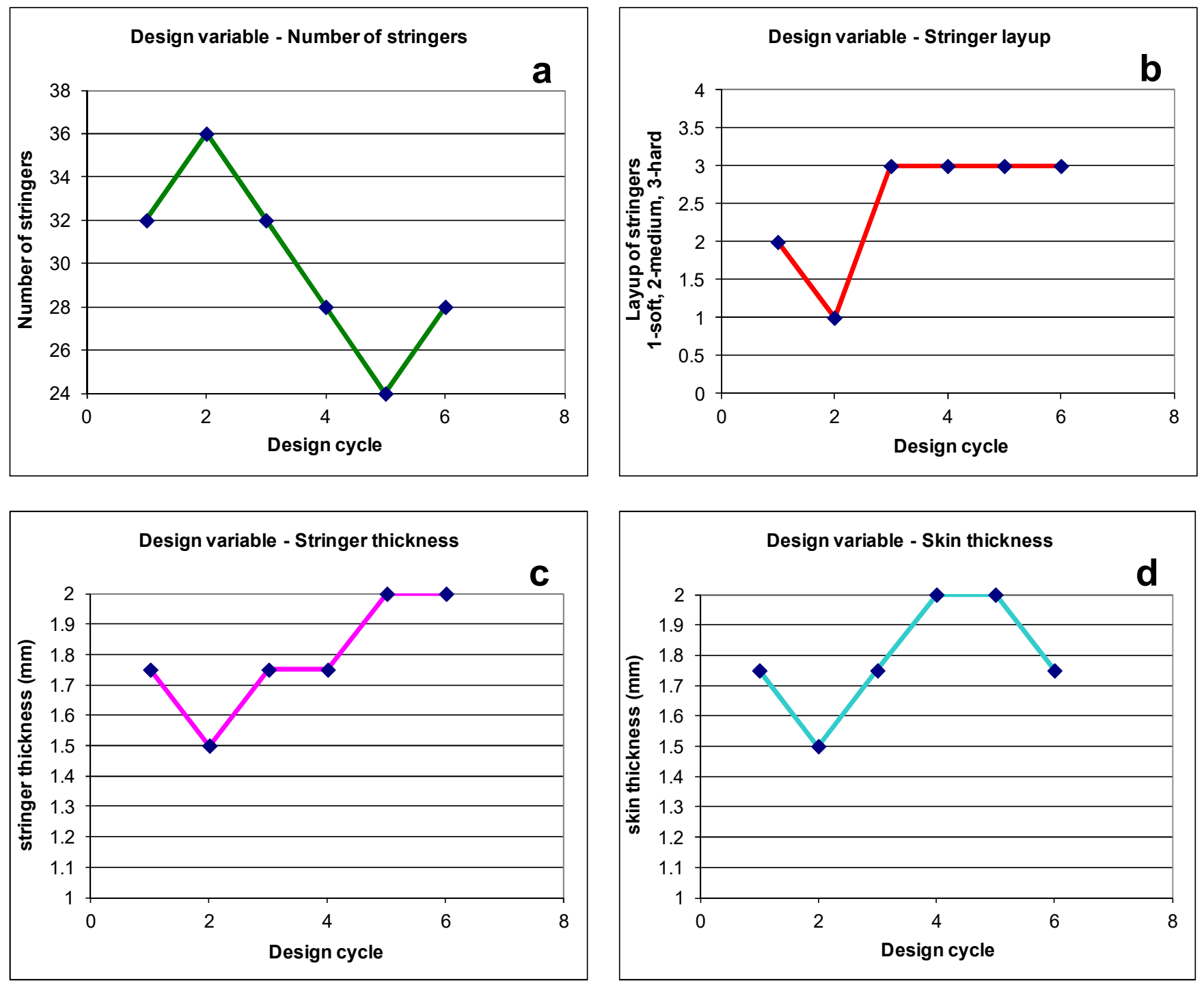
Fig.4

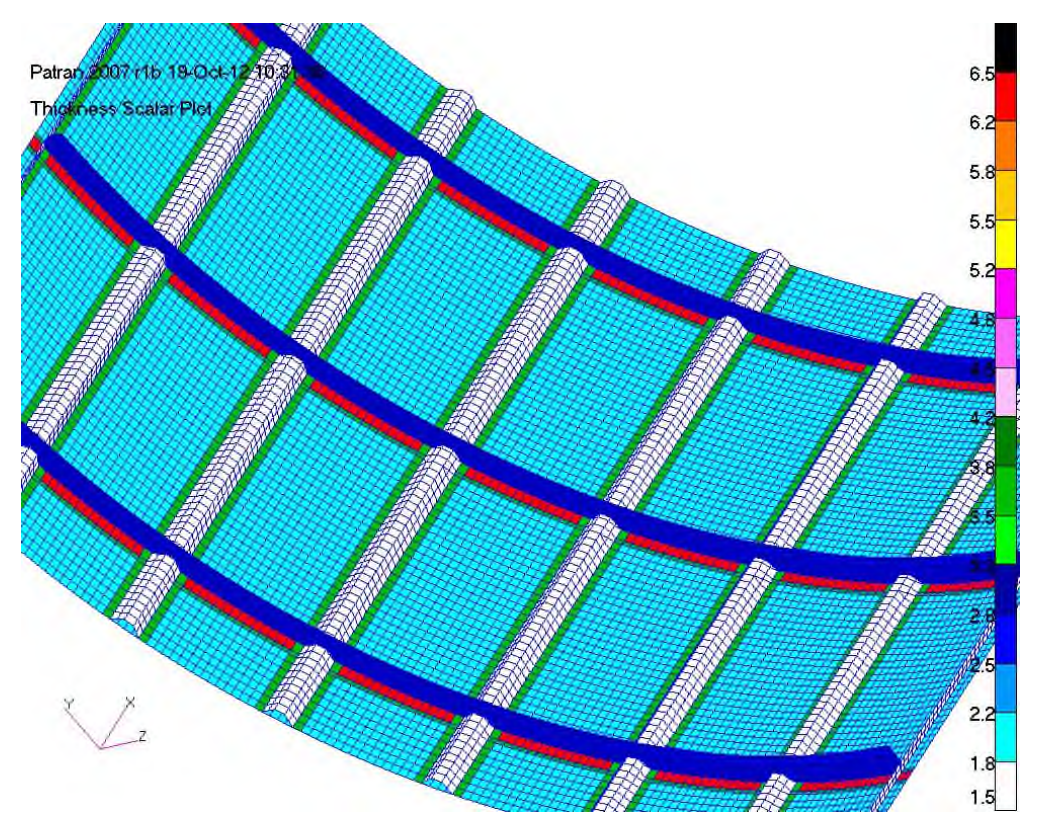


Fig.5

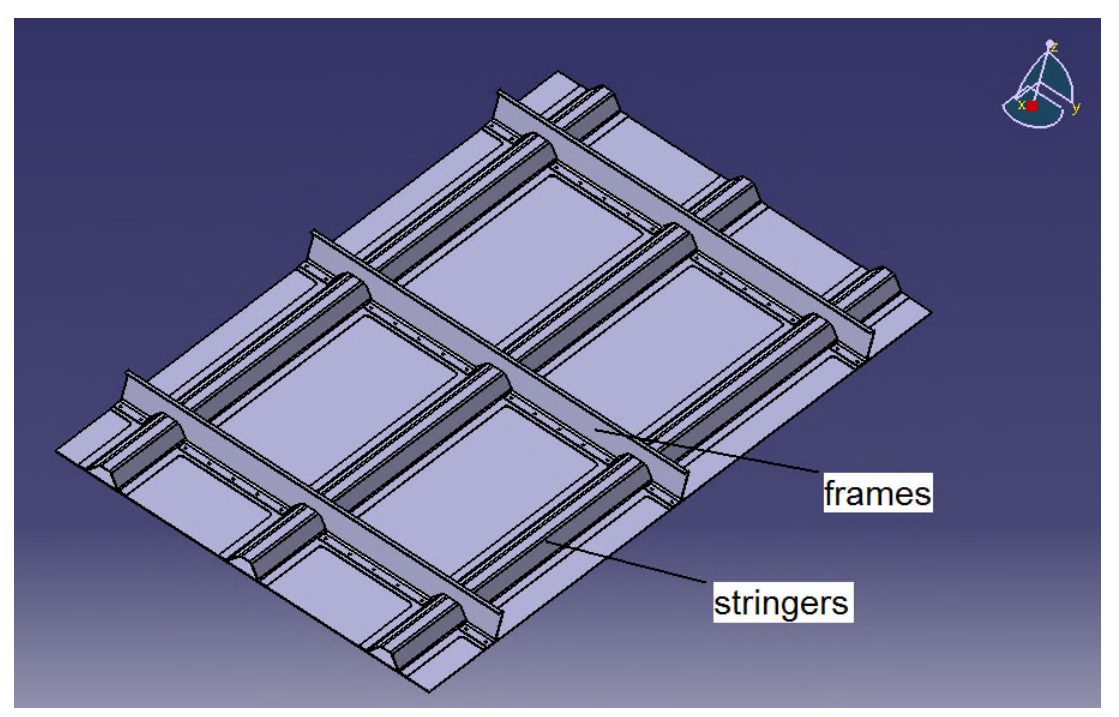


Fig.6

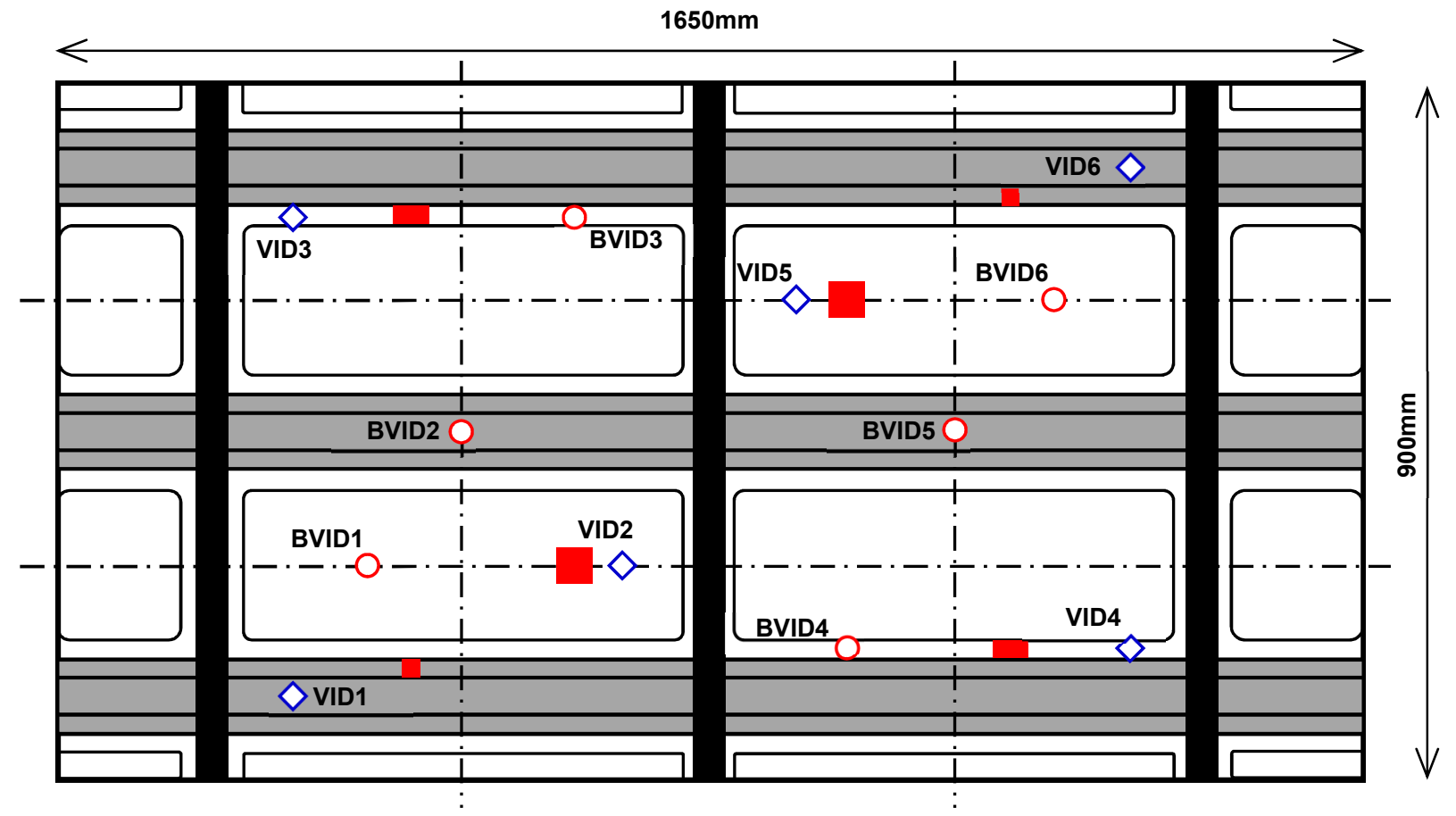

Stringer

Stiffener $O$ BVID location

$\diamond$ VID location

Artificial delamination A $\quad$ Artificial delamination B

Artificial delamination $\mathbf{C}$ 
Fig.7
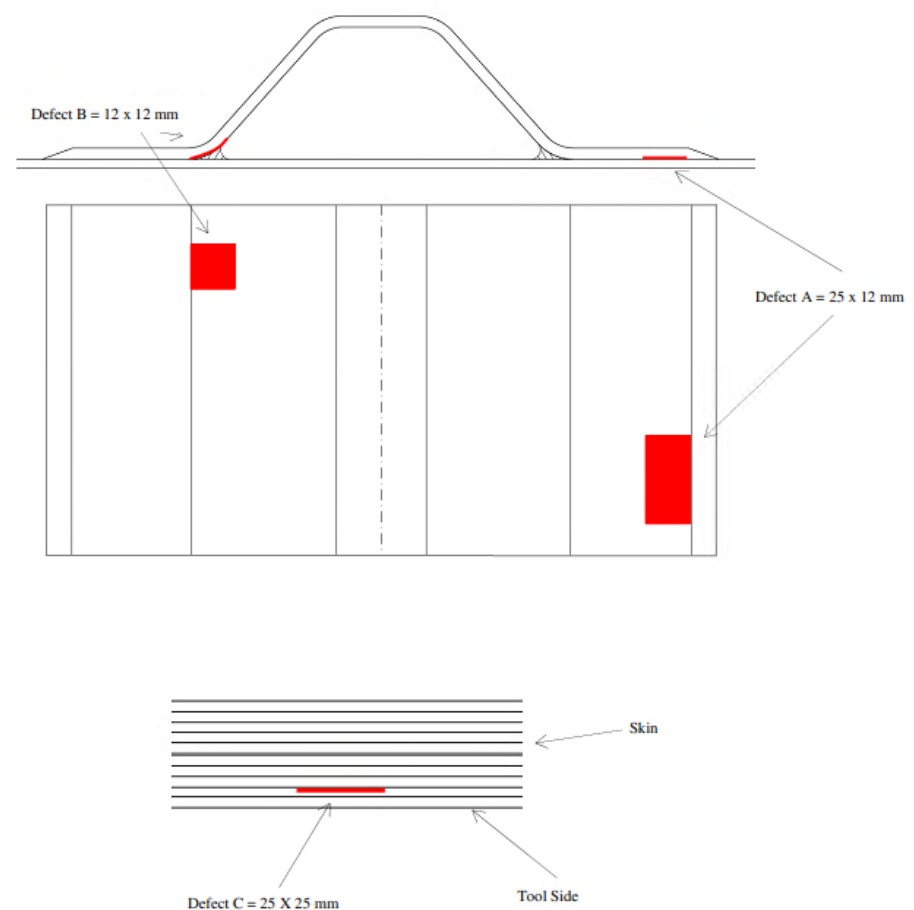
Fig.8

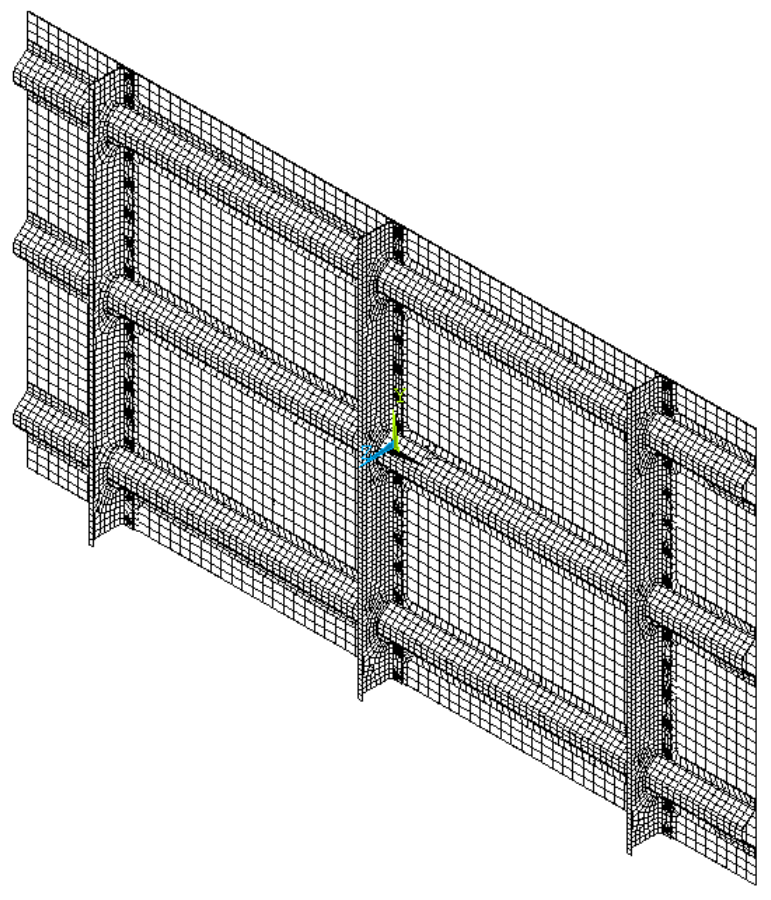


Fig.9

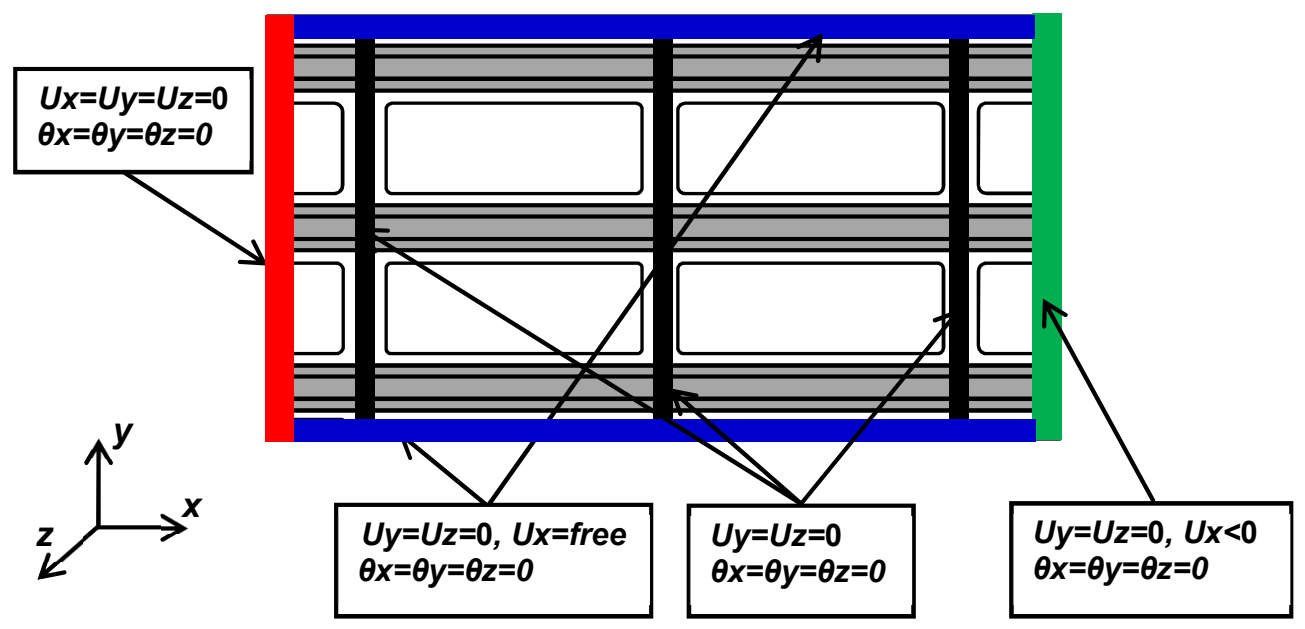


Fig.10

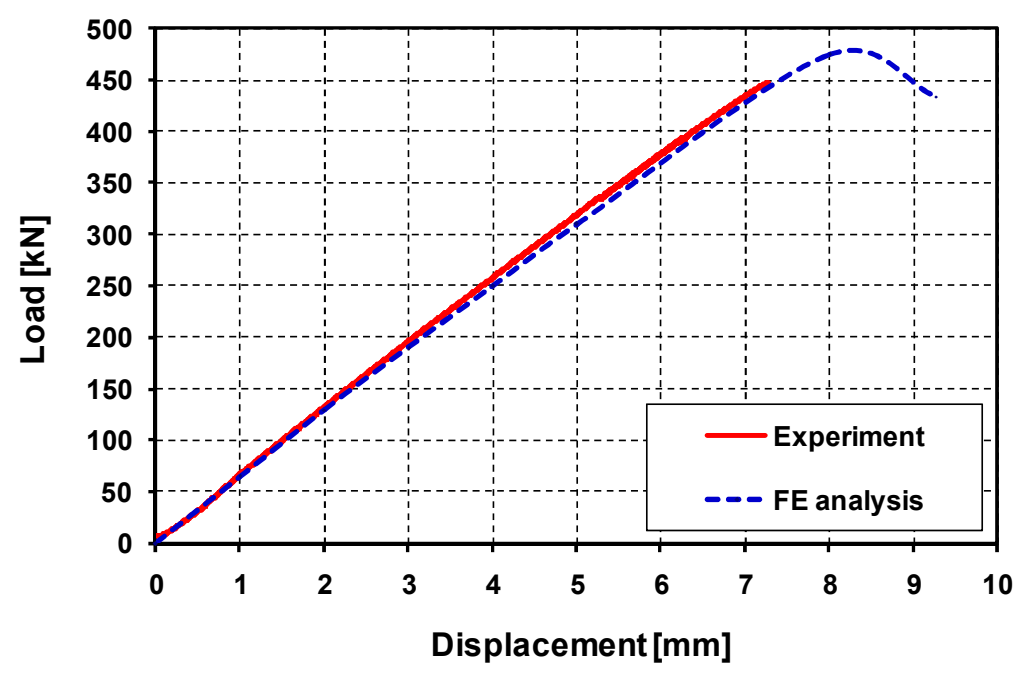


Fig.11

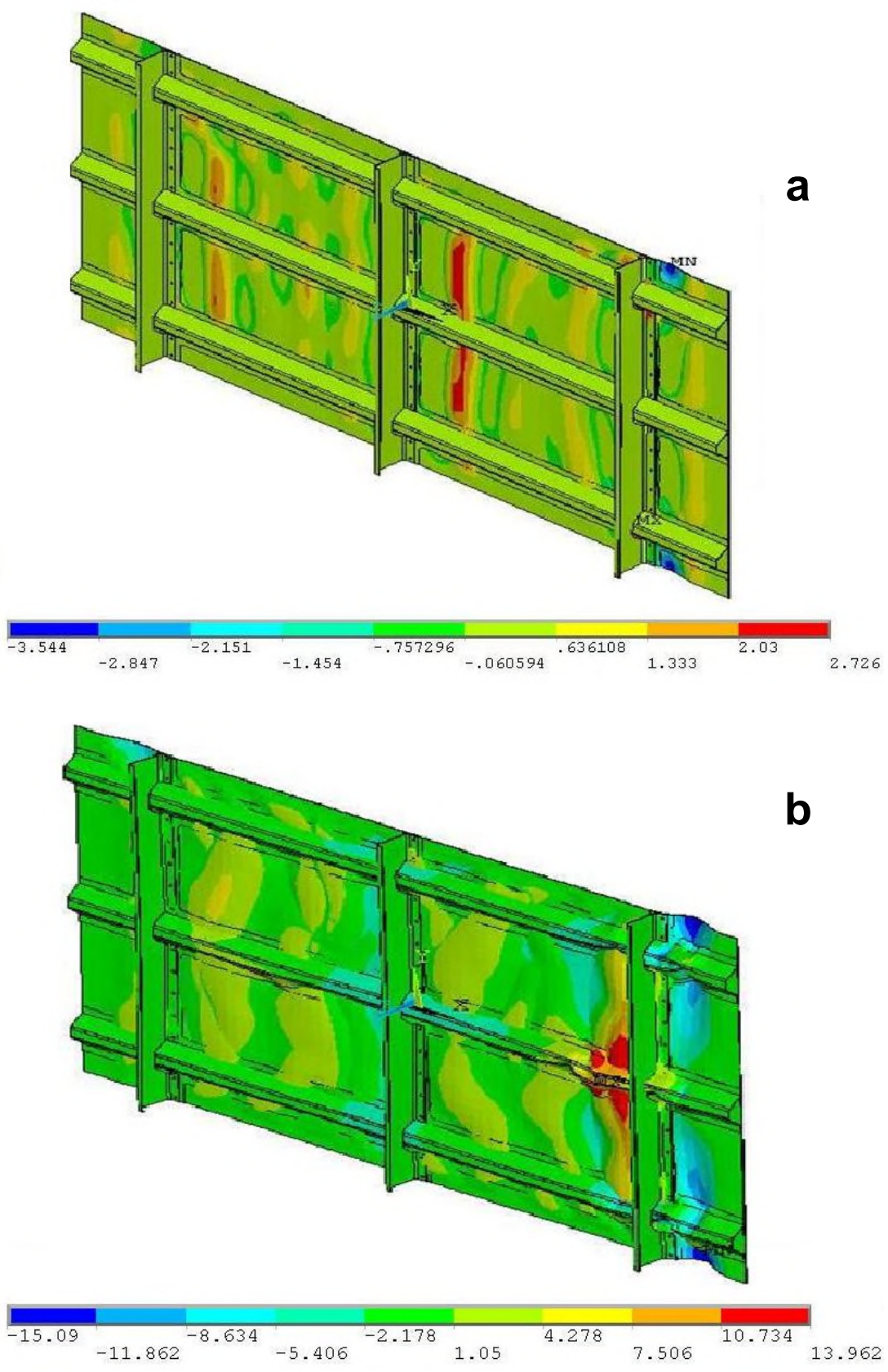


Fig.12

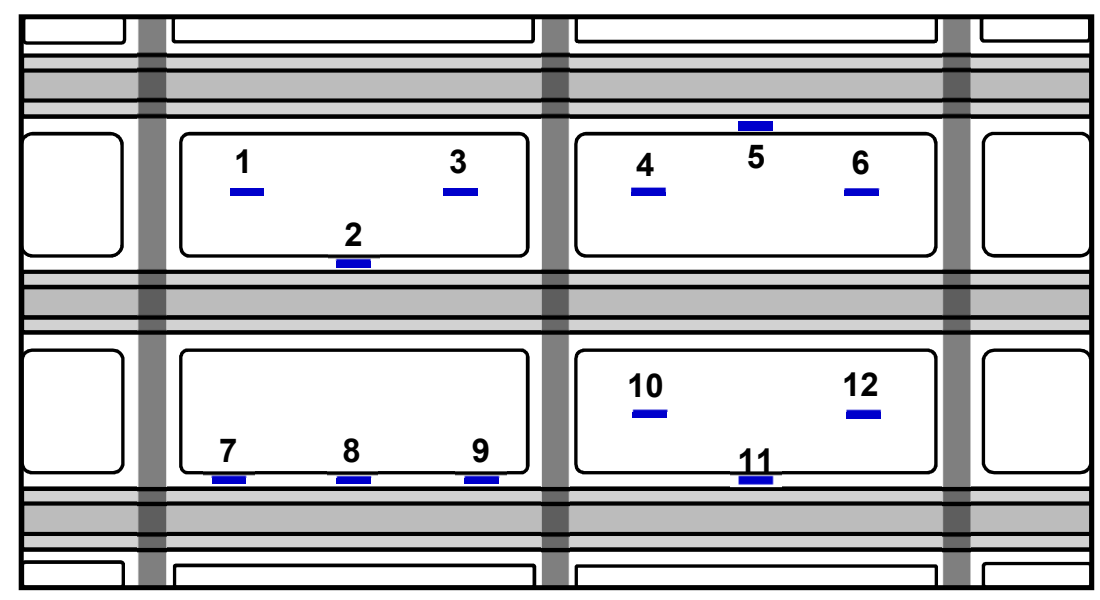


Fig.13

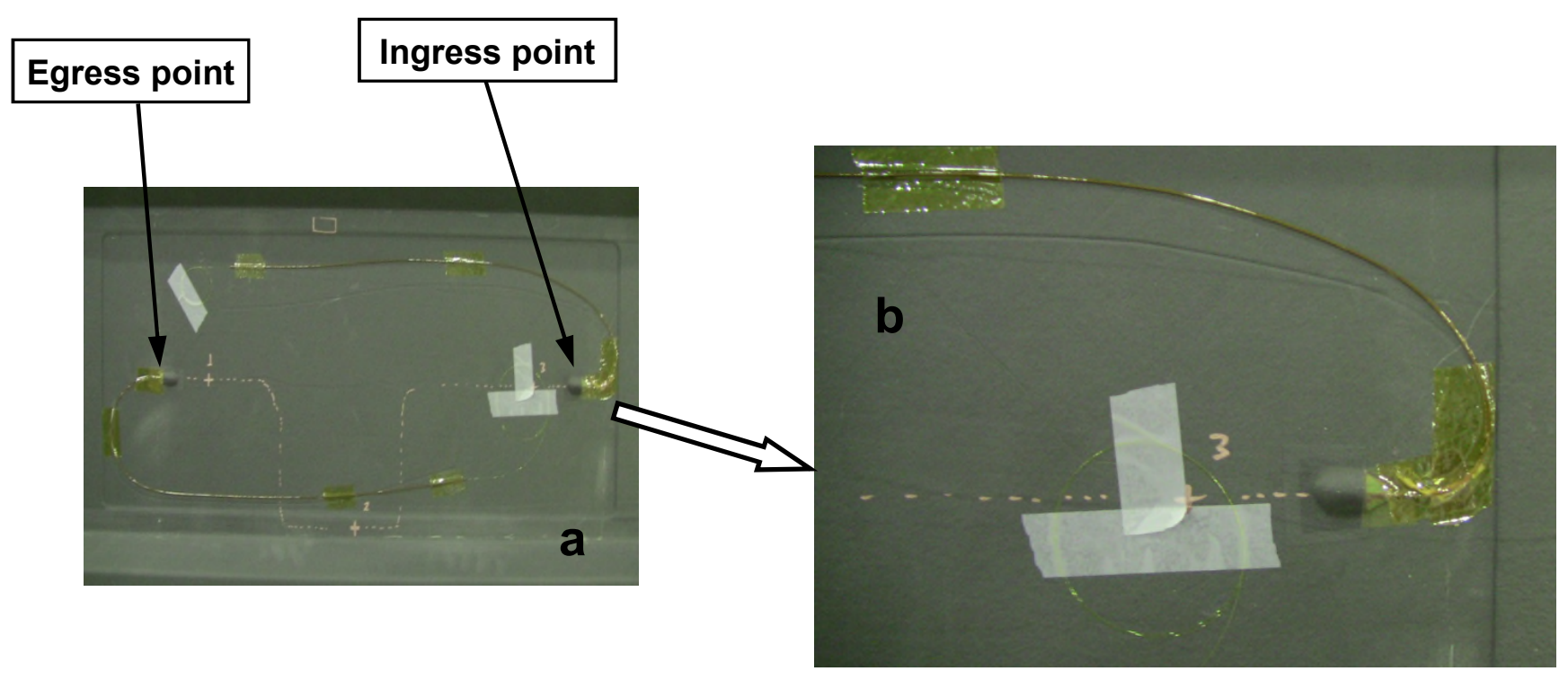


Fig.14
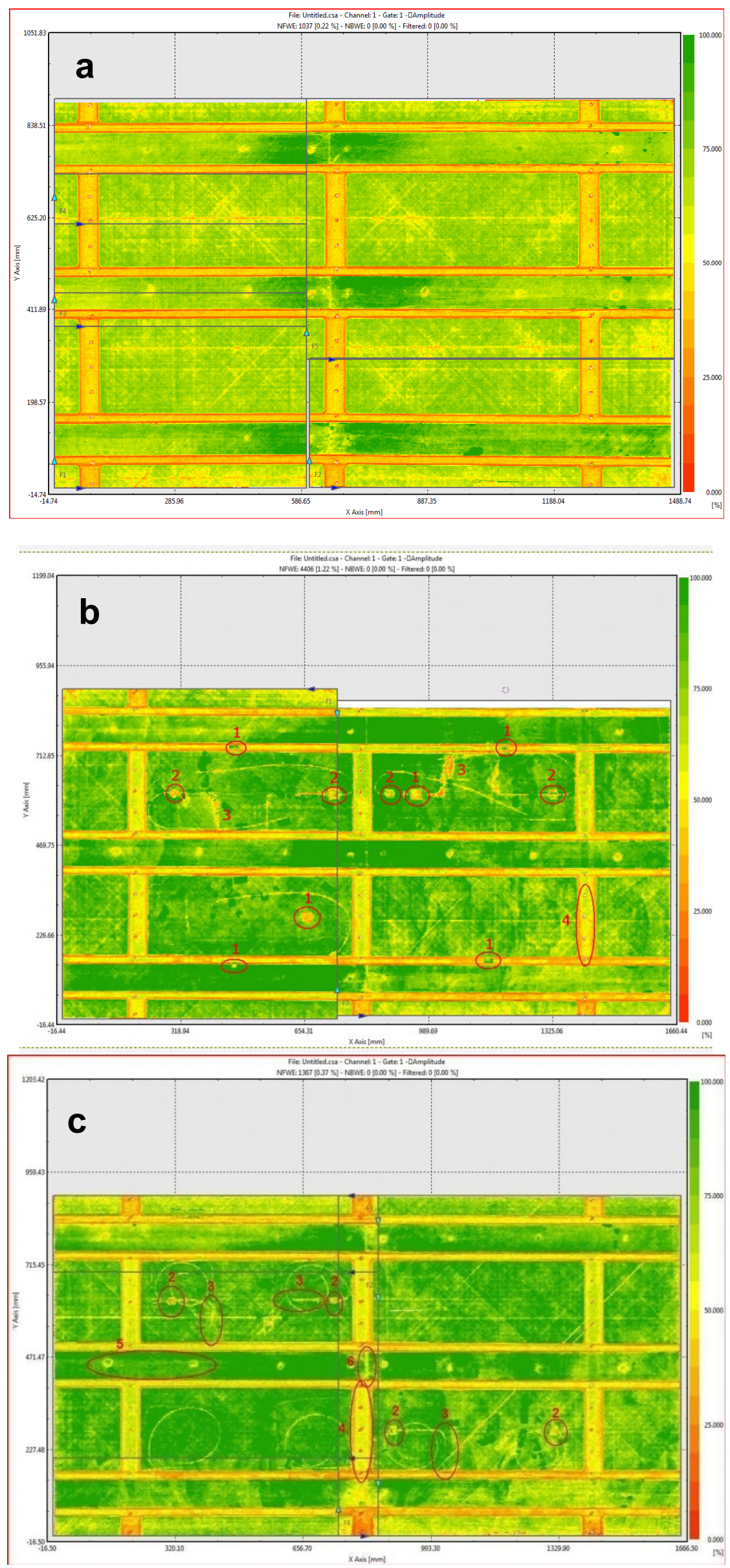
Fig.15

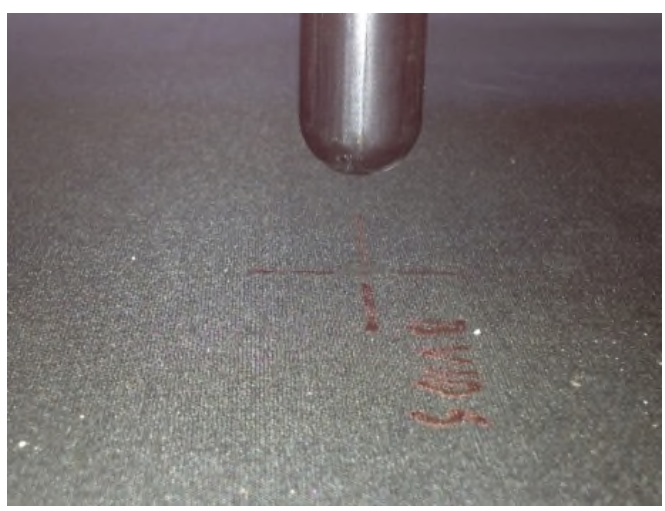


Fig.16

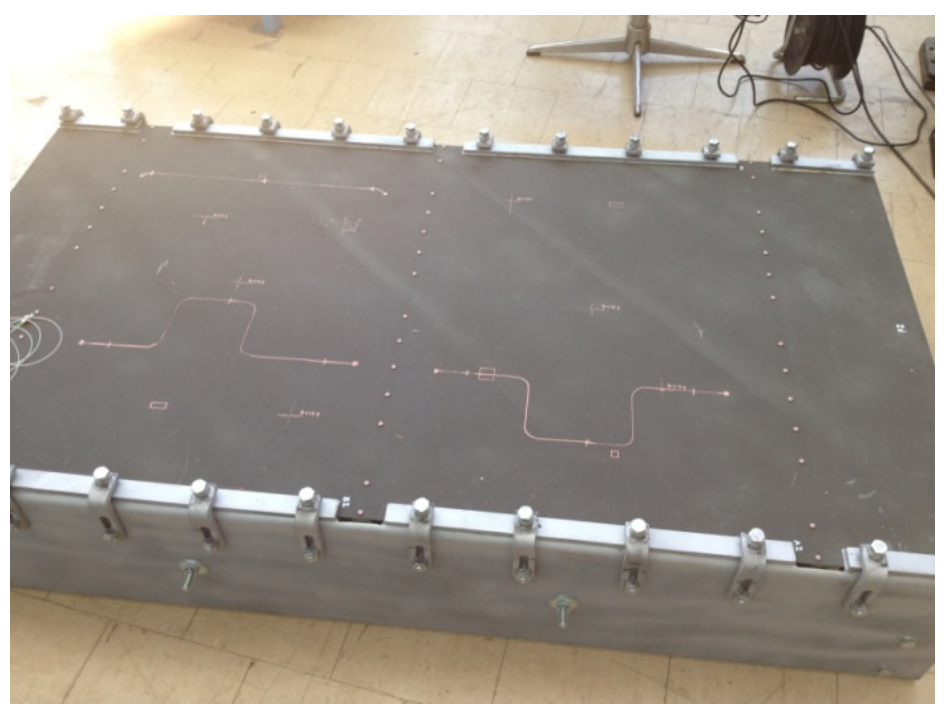


Fig.17
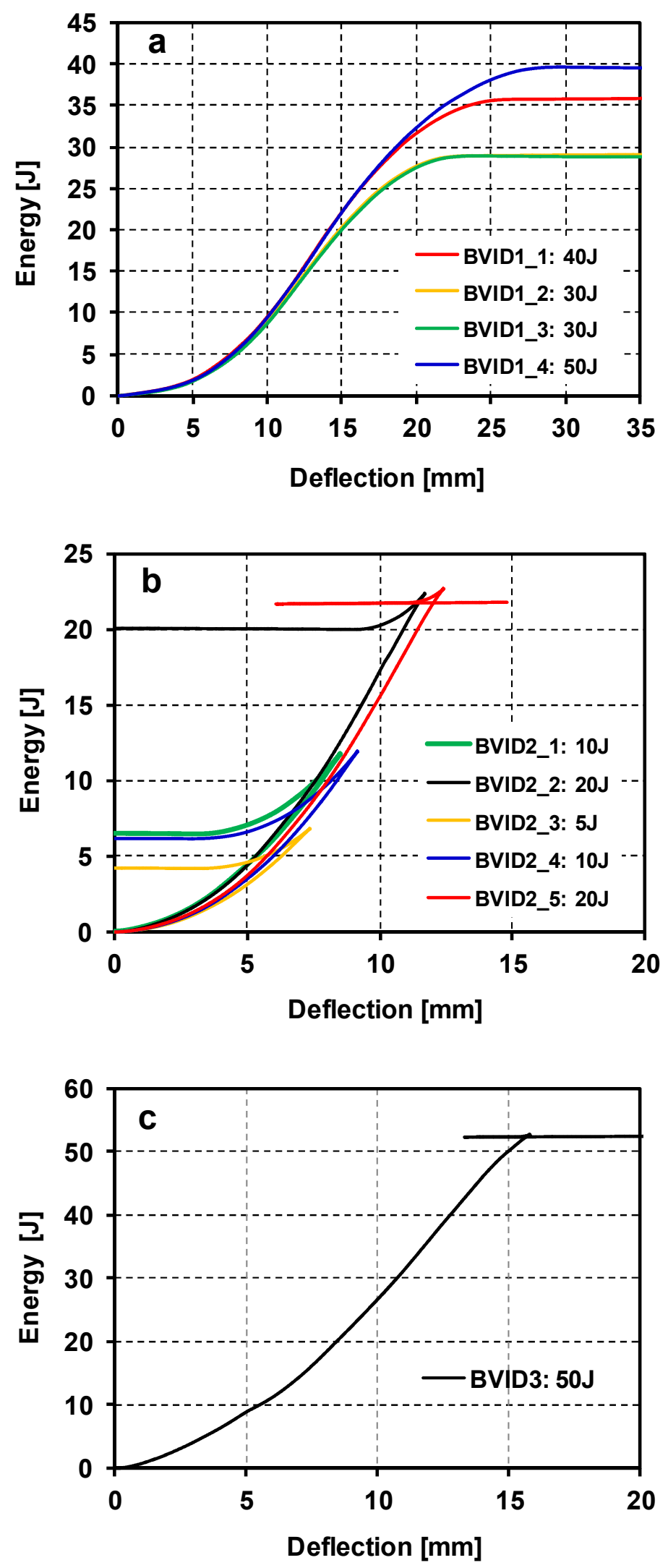
Fig.18
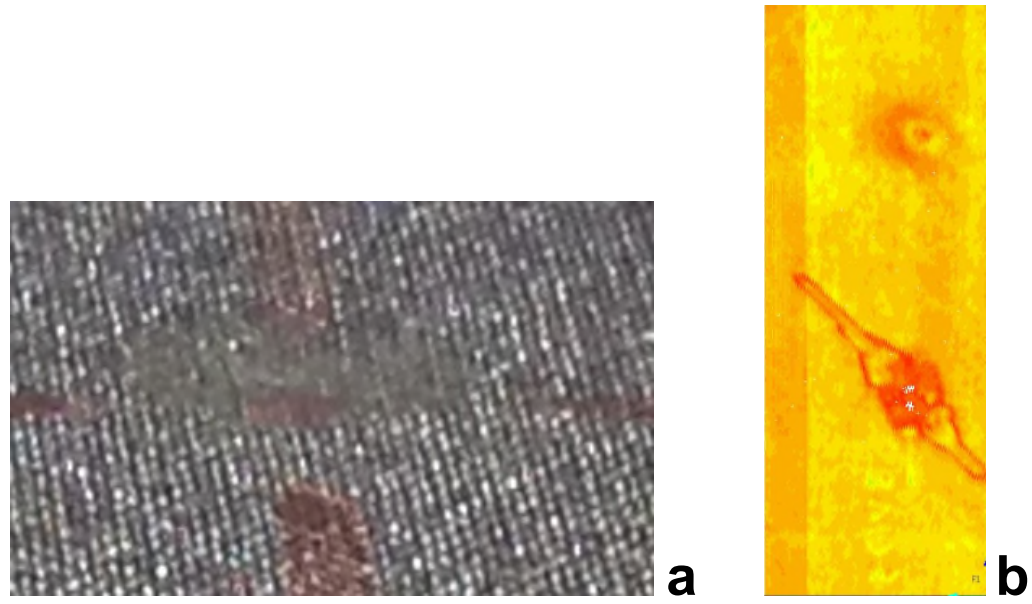
Fig.19

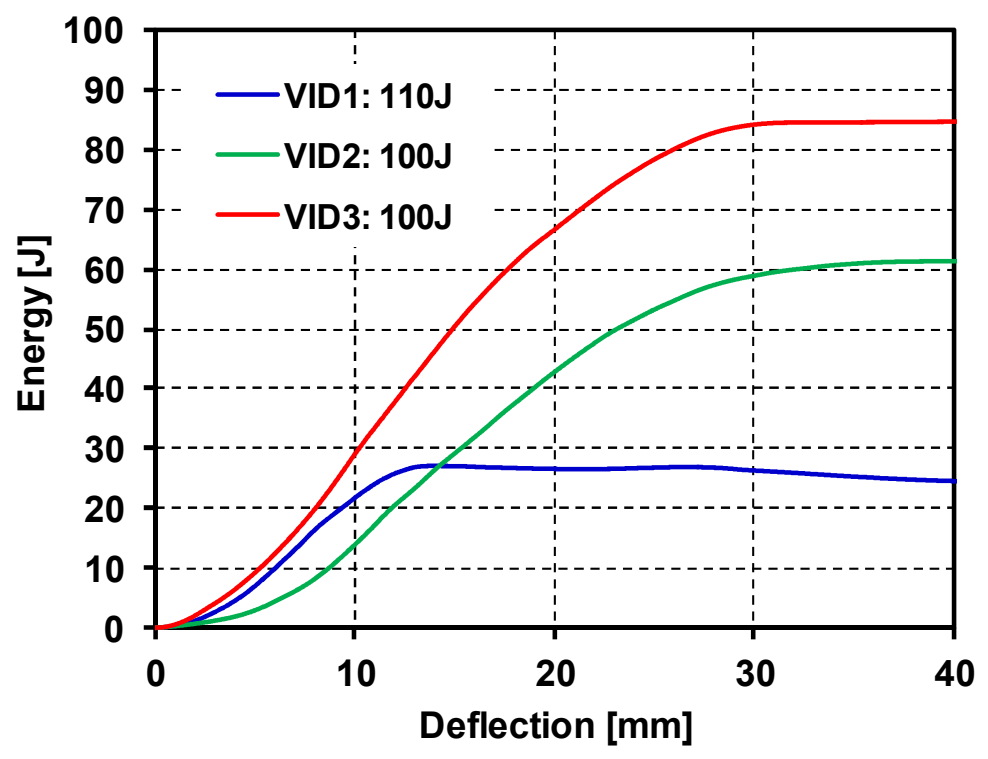


Fig.20
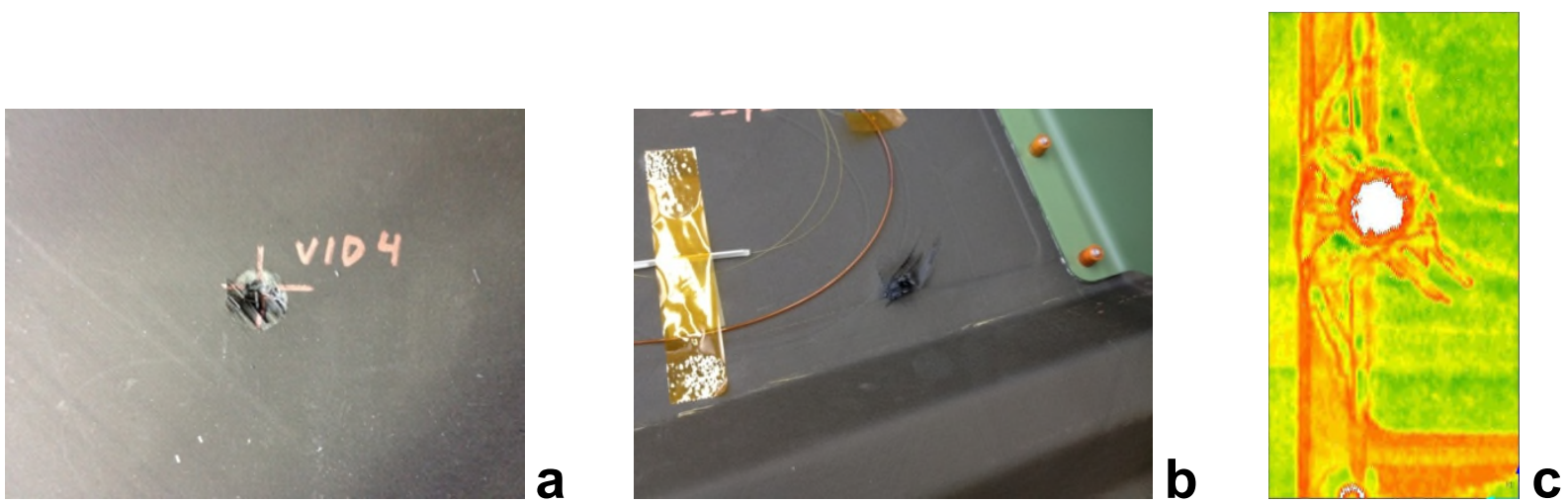
Table 1

Table 1: Demarcation of sensors for each panel.

\begin{tabular}{|c|c|c|c|}
\hline Sensor position & Panel_1 & Panel_2 & Panel_3 \\
\hline 1 & $\mathrm{x}$ & $\mathrm{x}$ & $\mathrm{x}$ \\
\hline 2 & $\mathrm{x}$ & $\mathrm{x}$ & $\mathrm{x}$ \\
\hline 3 & $\mathrm{x}$ & $\mathrm{x}$ & $\mathrm{x}$ \\
\hline 4 & $\mathrm{x}$ & $\mathrm{x}$ & \\
\hline 5 & $\mathrm{x}$ & $\mathrm{x}$ & \\
\hline 6 & $\mathrm{x}$ & $\mathrm{x}$ & \\
\hline 7 & $\mathrm{x}$ & $\mathrm{x}$ & $\mathrm{x}$ \\
\hline 8 & $\mathrm{x}$ & $\mathrm{x}$ & $\mathrm{x}$ \\
\hline 9 & $\mathrm{x}$ & $\mathrm{x}$ & $\mathrm{x}$ \\
\hline 10 & & & $\mathrm{x}$ \\
\hline 11 & & & $\mathrm{x}$ \\
\hline 12 & & & $\mathrm{x}$ \\
\hline
\end{tabular}


Strain and damage monitoring in CFRP fuselage panels using fiber Bragg grating sensors. Part I: Design, manufacturing and impact testing

Tserpes, Konstantinos I.

Elsevier

Tserpes KI, Karachalios V, Giannopoulos I, et al., (2014) Strain and damage monitoring in CFRP fuselage panels using fiber Bragg grating sensors. Part I: Design, manufacturing and impact testing. Composite Structures, Volume 107, January 2014, pp. 726-736

https://doi.org/10.1016/j.compstruct.2013.09.053

Downloaded from Cranfield Library Services E-Repository 